Full Paper

\title{
Die Optimierung des Weinausbaues von Cabernet Cortis
}

\section{The optimization of the winemaking of Cabernet Cortis}

\section{L'ottimizzazione della vinificazione del Cabernet Cortis}

\section{Christoph Patauner ${ }^{1}$, Ulrich Pedri ${ }^{1}$, Christof Sanoll ${ }^{1}$}

${ }^{1}$ Versuchszentrum Laimburg, Pfatten, Italien

\section{ABSTRACT}

Partially resistant grape varieties represent a possibility to achieve a successful harvest with low crop protection effort. However, the quality of the wines, sometimes is not satisfactory. The red variety Cabernet Cortis, is a valid alternative to an aging red cabernet type. The winemaking itself still needs to be optimized, as the variety has a very distinctive tannin component that needs to be rounded. Furthermore, the typical fruity components should be better interpreted. In this project, grapes of the variety Cabernet Cortis were subjected to different mash contact times and temperatures during fermentation.

\section{KEYWORDS}

Cabernet Cortis, winemaking Cabernet Cortis, Piwi winemaking, fungus resistant grape varieties winemaking

\section{CITE ARTICLE AS}

Patauner Christoph, Pedri Ulrich, Sanoll Christof et.al. (2020). The optimization of the winemaking of Cabernet Cortis. Laimburg Journal 02/2020

DOI: $10.23796 / \mathrm{LJ} / 2020.002$

\section{CORRESPONDING AUTHOR}

Christoph Patauner

Laimburg 6, Pfatten, I-39040 Auer (BZ), Italien

christoph.patauner@laimburg.it +390471969626 


\section{EINFÜHRUNG}

Die teilresistente Sorte Cabernet Cortis hat im Laufe der Sortenprüfung am Versuchszentrum Laimburg (Anbaueignung pilzwiderstandsfähiger Sorten) positive Ergebnisse geliefert und zwar sowohl bezüglich ihrer weinbaulichen Eigenschaften als auch bezüglich der Weincharakteristiken.

Teilresistente Rebsorten stellen eine Möglichkeit dar, mit geringem Pflanzenschutzaufwand zu einer erfolgreichen Ernte zu gelangen. Allerdings sind die weinqualitativen Eigenschaften dieser Sorten häufig nicht zufriedenstellend.

Die Sorte Cabernet Cortis stellt hingegen eine gültige Alternative zu einem alterungsfähigen Rotwein des Cabernet-Typs dar, wobei der Weinausbau selbst noch zu optimieren ist. Die Sorte weist eine sehr markante Gerbstoffkomponente auf, die es abzurunden gilt. Auch die typisch fruchtigen Komponenten gilt es besser zu interpretieren. Aus diesem Grund wurden Trauben der Sorte Cabernet Cortis verschiedenen Maischekontaktzeiten und Temperaturverläufen unterzogen.

\section{METHODEN}

Der Versuch war zeitlich in zwei Teile gegliedert. Zu Anfang des Projektes galt es zu verstehen, in welcher Variante die größten Möglichkeiten liegen, während im zweiten Teil eine Feinjustierung der vorteilhaftesten Variante stattfand. In den Jahren 2012, 2013 und 2015 wurden somit Trauben der Sorte Cabernet Cortis zu Wein verarbeitet. Tabelle 1 gibt einen Überblick über die ausgebauten Varianten.

Aufgrund von nicht ausreichend verfügbarer Traubenmaische wurden im Jahr 2012 lediglich von den Varianten „Gärtemperatur max. $20{ }^{\circ} \mathrm{C}$, Maischekontakt 4 Tage" und "Gärtemperatur max. $28{ }^{\circ} \mathrm{C}$, Maischekontakt Ausgärung" je eine Kellerwiederholung durchgeführt. Im Jahr 2013 wurden alle Varianten ohne Kellerwiederholung ausgebaut. Im Jahr 2015 wurden alle drei geprüften Varianten mit je einer Wiederholung im Keller ausgebaut. Die Variante „Mostgärung Rosè" wurde zwar in einem Versuchsjahr ausgebaut, man beschränkte sich allerdings auf das Sammeln von analytischen Daten. In Tabelle 2 sind die Mostanalysen der Ausgangsmaischen angeführt.

\section{ÖNOLOGIE}

Die Trauben wurden am entsprechenden Lesezeitpunkt von Hand geerntet und in Kisten zu 18 kg Fassungsvermögen in den Kellereibetrieb des Versuchszentrums Laimburg gebracht.

In jedem Versuchsjahr wurde die gesamte angelieferte Menge an Cabernet Cortis mit der Abbeermaschine des Typs CMA Lugana $1 R$ (Stundenleistung 4-6 $\mathrm{t} / \mathrm{h}$ ) abgebeert. Die gesamte Maische wurde in einer großen Wanne gesammelt und unter ständigem Vermischen auf Weithalskorbflaschen mit 34 I Fassungsvermögen aufgeteilt, um eine identische Ausgangsmaische zum Vergleich der Varianten zu erzielen.

Die Maische wurde mit 20 mg/l Kaliummetabisulfit (E 224) behandelt. Es wurde je Glasballon eine Mostprobe entnommen und diese auf die gängigen Mostparameter untersucht. Die Varianten mit 6 Tagen Kaltmazeration kamen für diesen Zeitraum in die Kühlzelle bei $4{ }^{\circ} \mathrm{C}$.

Unabhängig ob mit oder ohne Kaltmazeration wurden die Maischen vor Hefebeimpfung auf $20^{\circ} \mathrm{C}$ erwärmt und in Folge mit 25 $\mathrm{g} / \mathrm{hl}$ Trockenreinzuchthefe des Typs Saccharomyces cerevisiae var. cerevisiae (Levuline $B R G^{\mathrm{T}}{ }^{\mathrm{M}}$ ) geimpft. Die Temperaturführung während der alkoholischen Gärung erfolgte über ein automatisches temperaturgesteuertes System. Die Mazerationsdauer ist aus der Variantenbeschreibung zu entnehmen. Der 1. Abstich erfolgte belüftend über ein Kupfersieb ohne Schwefelung (E 224). Der Presswein (0,5 bar für $10 \mathrm{~min}$ ) wurde zu $100 \%$ dem Wein beigemengt. Die malolaktische Gärung erfolgte ohne Inokulation von Milchsäurebakterien bei $20^{\circ} \mathrm{C}$. Nach Abschluss der malolaktischen Gärung (Äpfelsäure $<0,1 \mathrm{~g} / \mathrm{l}$ ) erfolgte ein Abzug und eine Zugabe von $30 \mathrm{mg} / \mathrm{l}$ Kaliummetabisulfit (E224).

Die Lagerung der Weine erfolgte bei einer Temperatur zwischen 14 und $18{ }^{\circ} \mathrm{C}$. Während der Weinlagerung wurde ein Gehalt an freier schwefeliger Säure von $20-25 \mathrm{mg} / \mathrm{l}$ eingestellt und überwacht. Die Füllung erfolgte in 0,5 I Glasflaschen nach unmittelbar vorhergehender Vor-, Blank- und Sterilfiltration $(0,45 \mu \mathrm{m})$.

Es wurden auf Most und Wein folgende chemische Analysen für folgende Inhaltsstoffe durchgeführt:

- $\mathrm{pH}-$ Wert Most und Wein (FOSS ${ }^{\circledR}$, Wine$S_{C a n}{ }^{T M}$, Methodenbuch 5.04pp45
- Gesamtsäure g/l Most und Wein (FOSS ${ }^{\circledast}$ WineScan ${ }^{T M}$, Methodenbuch 5.04pp45)

- Zuckergradation ${ }^{\circ} \mathrm{KMW}$ im Most (FOSS ${ }^{\circledR}$, WineScan ${ }^{T M}$, Methodenbuch 5.04pp45)

- Flüchtige Säure im Wein g/l (FOSS ${ }^{\circledR}$, Wine$S c a n^{T M}$, Methodenbuch 5.04pp45)

- Restzucker g/l (FOSS $^{\circledR}$, WineScan ${ }^{T M}, \mathrm{Me}$ thodenbuch 5.04pp45)

- Gesamtextrakt g/l (FOSS ${ }^{\circledR}$, WineScan ${ }^{\text {TM }}$ Methodenbuch 5.04pp45)

- Anthocyane mg/l im Wein (Methodenbuch 5.04pp02)

- Weinsäure g/l im Wein (Methodenbuch 5.04pp52)

- Milchsäure g/l im Wein (Methodenbuch 5.04pp53)

- Gesamtpolyphenole mg/l im Wein (Methodenbuch 5.04pp17)

- Alkohol \% vol im Wein (Methodenbuch 5.04pp09)

- Methanol g/l im Wein (Methodenbuch 5.04pp03)

- Farbindizes 420/520/620 (Methodenbuch 5.04pp39)

\section{SENSORIK}

Alle Weine wurden rund 18 Monate nach der Einkellerung einer sensorischen Prüfung unterzogen. Die sensorisch zu diskriminierenden Kriterien für den Jahrgang 2012 sind in Tabelle 3 zu sehen, jene für den Jahrgang 2013 in Tabelle 4 und jene für den Jahrgang 2015 in Tabelle 5.

Für die sensorische Charakterisierung wurde ein Verkostungsprofil nach Weiß 1972 [1] (modifiziert - nicht publiziert) verwendet (Abb. 1). Um die Urteilssicherheit der einzelnen Panelmitglieder zu überprüfen wurde das Verfahren nach Kobler 1996 [2] angewandt. Dabei wurde für jeden einzelnen Parameter die Kontrollalgorithmen durchgerechnet. Das Verkosterpanel setzte sich aus Technikern, Beratern und Fachleuten aus Weinbau und Kellerwirtschaft zusammen. Für die Auswertung der erhobenen Daten wurden die Programme Microsoft ${ }^{\circledR}$ Office Excel 2013 von CMicrosoft Corporation 1993-2001, für die statistische Auswertung IBM ${ }^{\circledR}$ SPSS $^{\circledR}$ Statistics Version 20 Release 20.0.0 für varianzanalytische Mittelwertvergleiche (Oneway ANOVA) oder multivarianter Varianzanalyse (Manova). Als Post-HocTest wurde Tukey-B mit Signifikanzniveau 0,05 mit SPSS gerechnet. 


\section{ERGEBNISSE AUS DEN JAHREN 2012 UND 2013}

Nach den Verkostungen der Weine der Jahrgänge 2012 und 2013 in den Jahren 2014 und 2015 wurde versucht, ein erstes Zwischenergebnis zu formulieren, um sich auf die einflussreichsten Varianten zu fokussieren (Abb. 2, Abb. 3, Abb. 4, Abb. 5).

\section{ZWISCHENDISKUSSION}

Vergleicht man die Varianten mit Kaltmazeration und ohne Kaltmazeration, so ergeben sich, sensorisch gesehen, mehr oder weniger dieselben Weine. Der Einfluss von Kaltmazeration auch in Hinblick auf die Fruchtigkeit der Weine scheint also vernachlässigbar zu sein (Abb. 2). Auch beim Vergleich zwischen kühlen und warmen Gärtemperaturen ist der große erwartete Unterschied ausgeblieben. Die Varianten mit maximal $20^{\circ} \mathrm{C}$ haben zwar ein etwas runderes Tannin, aber vor allem in Punkto Fruchtigkeit der Weine nicht den erwarteten Vorteil gegenüber den wärmer vergorenen Varianten (Abb. 3). Die größten Unterschiede schienen in der Maischekontaktzeit zu liegen. Weine mit kurzer Maischekontaktzeit sind im Eindruck harmonischer, da sie vor allem eine leichtere Gerbstoffstruktur aufweisen und die Gerbstoffe sich eindeutig runder und weniger bitter präsentieren. Auch in einer Hauptkomponentenanalyse lassen sich die kurzen Gärvarianten eindeutig von den langen Gärvarianten abtrennen (Abb. 5).

Aus diesem Grund wurde im Versuchsjahr 2015 versucht, anhand von unterschiedlich langer Maischekontaktzeit, den Optimal-Bereich zwischen Maischekontaktzeit und analytisch messbaren Gesamtpolyphenolen auszuloten.

\section{ERGEBNISSE 2015}

Mit den Versuchsvarianten des Jahrgangs 2015 wurde eine Präzisierung im Umgang mit den Gerbstoffen von Cabernet Cortis erreicht. Der Gehalt an Anthocyanen im Wein verändert sich mit zunehmender Maischekontaktzeit. Der Gehalt an Anthocyanen nimmt in den ersten Tagen der Gärung zu und baut sich dann mit zunehmender Maischekontaktzeit wieder ab (Abb. 6).

\section{ZUSAMMENFASSUNG}

Bei teilresistenten Rebsorten erzielt man meist mit geringem Aufwand an Pflanzenschutzmitteln eine erfolgreiche Ernte. Oft sind aber die weinqualitativen Eigenschaften dieser Rebsorten nicht befriedigend.

Eine gültige Alternative eines alterungsfähigen Cabernet-Typs stellt die Rebsorte Cabernet Cortis dar. Allerdings ist die Weinbereitung von Cabernet Cortis noch zu optimieren. Vor allem die sehr markante Gerbstoffkomponente gilt es abzurunden sowie die fruchtigen Komponenten besser zu interpretieren. Darum wurden Trauben der Rebsorte Cabernet Cortis verschiedenen Maischekontaktzeiten und Temperaturverläufen unterzogen.

\section{RIASSUNTO}

Varietà, le quali sono resistenti contro malattie fungine fino ad un certo grado ci danno la possibilità di ottenere un raccolto sano con un ridotto utilizzo di prodotti fitosanitari. Tuttavia, le caratteristiche qualitative dei vini rappresentano ancora un limite.

Una di queste „Piwi” è la varietà Cabernet Cortis, la quale rappresenta una valida alternativa ad un vino rosso atto all'invecchiamento. La vinificazione in sé è ancora migliorabile, in quanto la varietà presenta un'importante componente tannica e aromatica.

Lo scopo di questo progetto era quello di dare un giudizio sulla vinificazione al fine di aumentare la qualità dei vini.

Der Gehalt an Gesamtpolyphenolen steigt mit zunehmender Maischekontaktzeit deutlich an (Abb. 7).

Fast identisch mit dem Gehalt an Gesamtpolyphenolen steigt auch der Gesamtextrakt im Wein an (Abb. 8, Abb. 9).

Auffallend ist dabei, dass mit zunehmendem Gehalt an Gesamtpolyphenolen die Harmonie im Wein abnimmt (Abb. 10, Abb. 11, Abb. 12).

Der Gesamteindruck des Weines nimmt mit zunehmender Maischekontaktzeit tendenziell ab, ebenso die Harmonie. Signifikante Unterschiede waren im Versuchsjahr 2015 allerdings nicht festzustellen (Abb. 13).

\section{DISKUSSION}

Die Ergebnisse haben gezeigt, dass bei der Rebsorte Cabernet Cortis eine eher kurze Maischekontaktzeit angestrebt werden sollte. Die Weine aus Gärungen mit kurzer
Maischekontaktzeit sind zwar tendenziell etwas weniger vielseitig, dafür aber fruchtiger und präsentieren sich aufgrund einer weniger ausgeprägten Gerbstoffkomponente harmonischer. Bei der Variante „5 Tage Maischegärung " ist der Gesamtextrakt signifikant nierdiger als in den beiden anderen Varianten. Allerdings stehen die Weine in Punkto Gesamteindruck auf derselben Ebene. Je länger die Maischekontaktzeit in der Gärung anhält, desto höher steigen auch der Gehalt an Flüchtiger Säure und der Gehalt an Methanol an (Tab. 6, Tab. 7, Tab. 8).

\section{DANKSAGUNG}

Wir danken Herrn Werner Pichler, Betriebsleiter der Anlage „Piglon“ für die gute Zusammenarbeit. Weiters bedanken wir uns bei allen Versuchsweinverkostern für ihren unentgeltlichen und wertvollen Dienst. 


\section{REFERENCES}

[1] Weiss J., Willisch E., Knorr D. et al. (1972). Ergebnisse von Untersuchungen bezüglich der differenzierten Wirkung einer sensorischen bewertenden Prüfmethode gegenüber einer sensorischen Rangordnungs-Prüfmethode am Beispiel von Apfelsaft und Birnennektar. Confructa 17 (4/5), 237-250.
[2] Kobler A. (1996). La valutazione sensoriale dei vini ed il controllo degli assaggiatori mediante l'uso di schede di degustazione non strutturate. Rivista di Viticoltura e di Enologia 49 (4), 3-18. 


\section{ANHANG 1: ABBILDUNGEN}

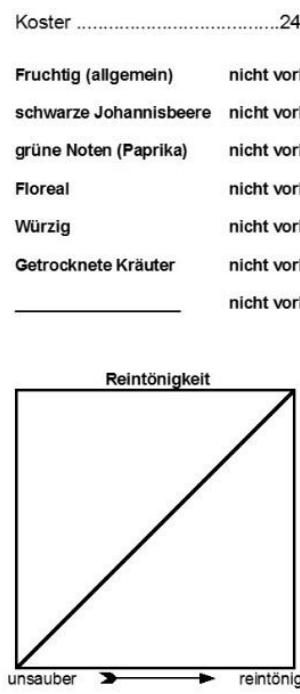

24.03.2017 Gruppe A

Glasnummer 1
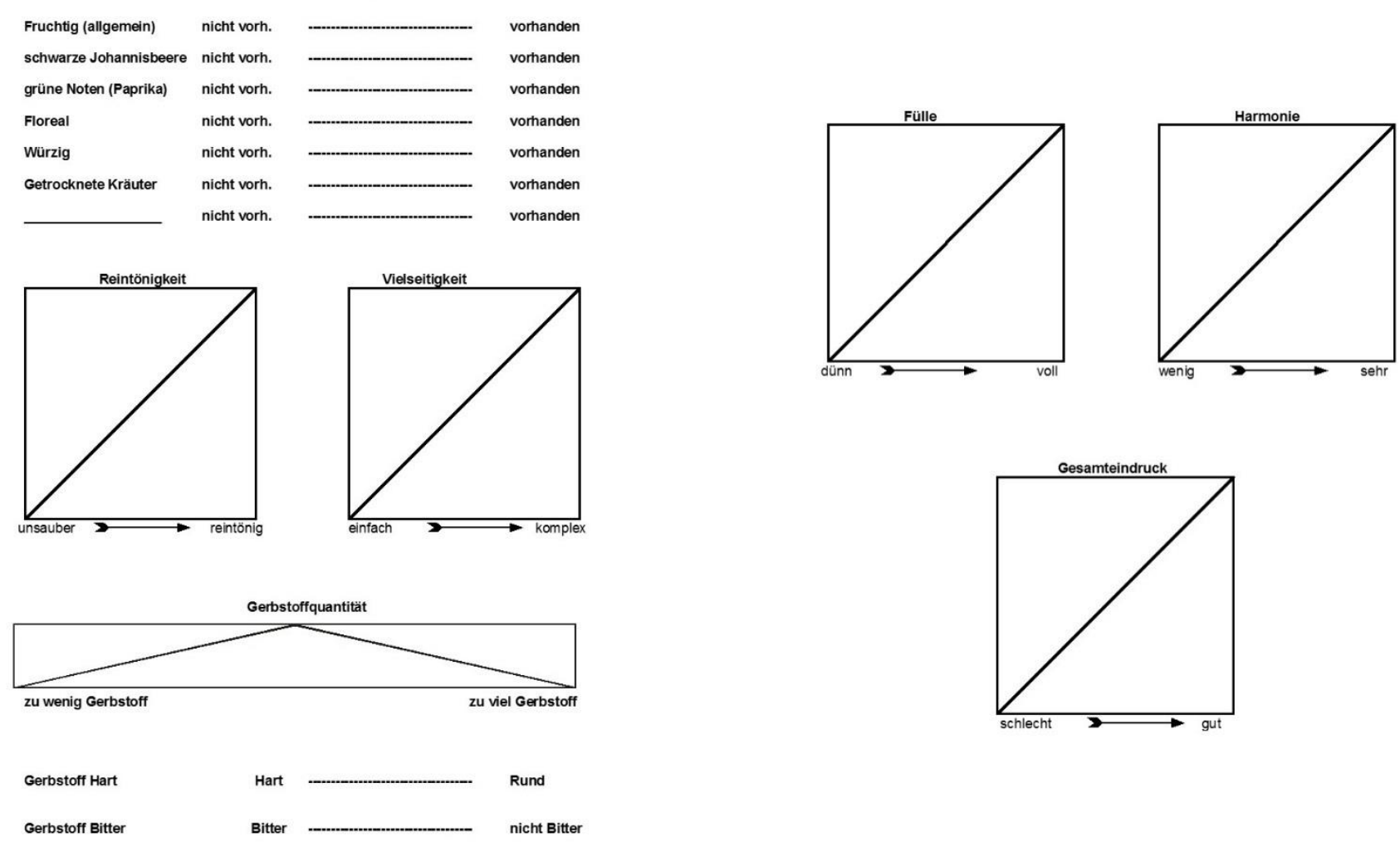

Abb. 1: Verkostungsbogen für sensorische Profilanalyse // Tasting sheet for sensory profile analysis. 


\section{Comparison: without cold maceration - cold maceration}

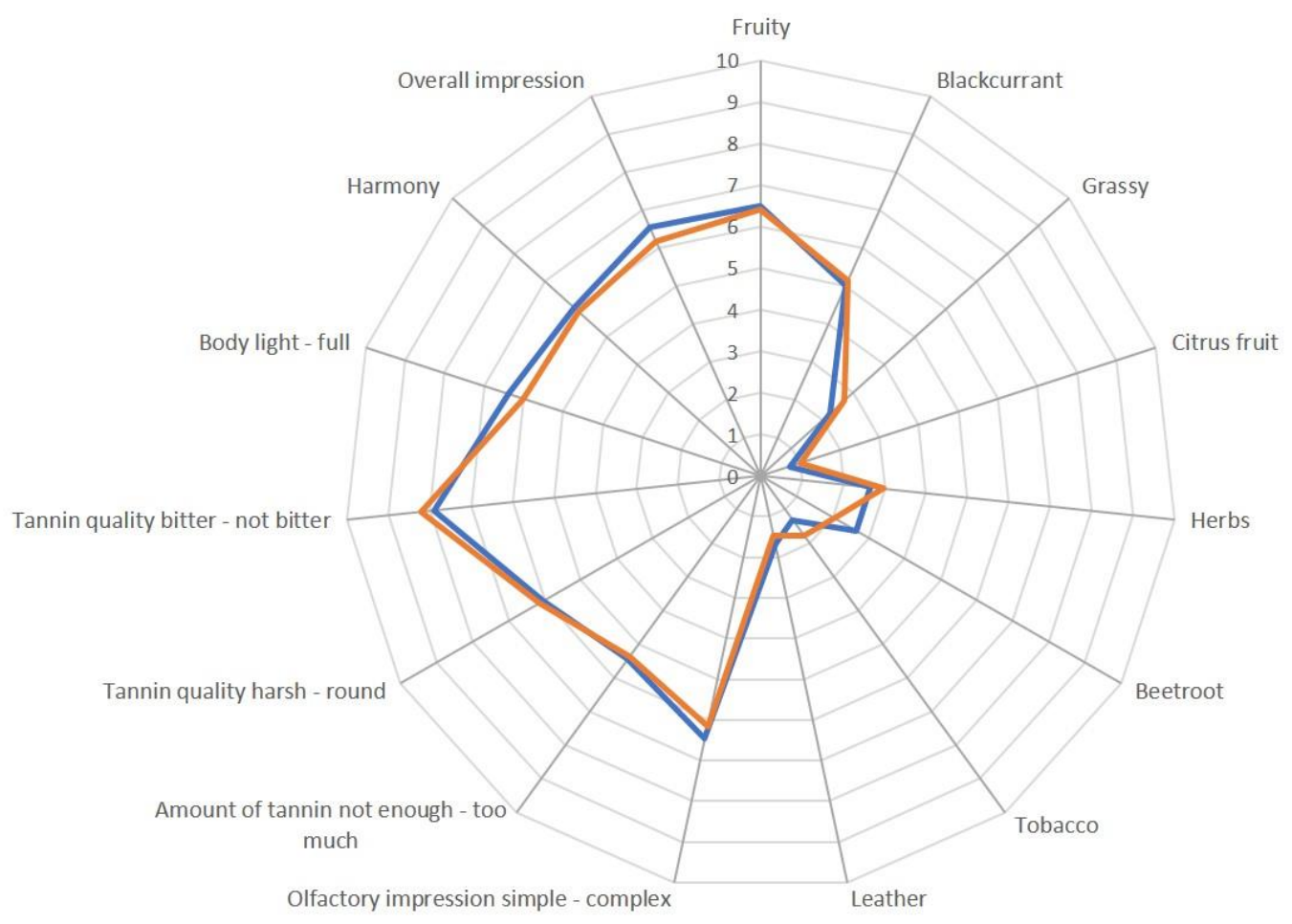

— cold maceration —without cold maceration

Abb. 2: Spinnennetzdiagramm zur sensorischen Beschreibung Jahrgang 2012 und 2013; Vergleich aller Varianten Kaltmazeration und ohne Kaltmazeration. ${ }^{*} \mathrm{p} \leq 0.05 ;^{* *} \mathrm{p} \leq 0.01 ; * * \mathrm{p} \leq 0.001 / /$ Radar chart of the sensorial description vintage 2012 and 2013; comparison of al variants cold maceration and without cold maceration. ${ }^{*} p \leq 0.05 ; *{ }^{*} p \leq 0.01 ; * * p \leq 0.001$. 


\section{Comparison: cool fermentation temperature - warm fermentation temperature}

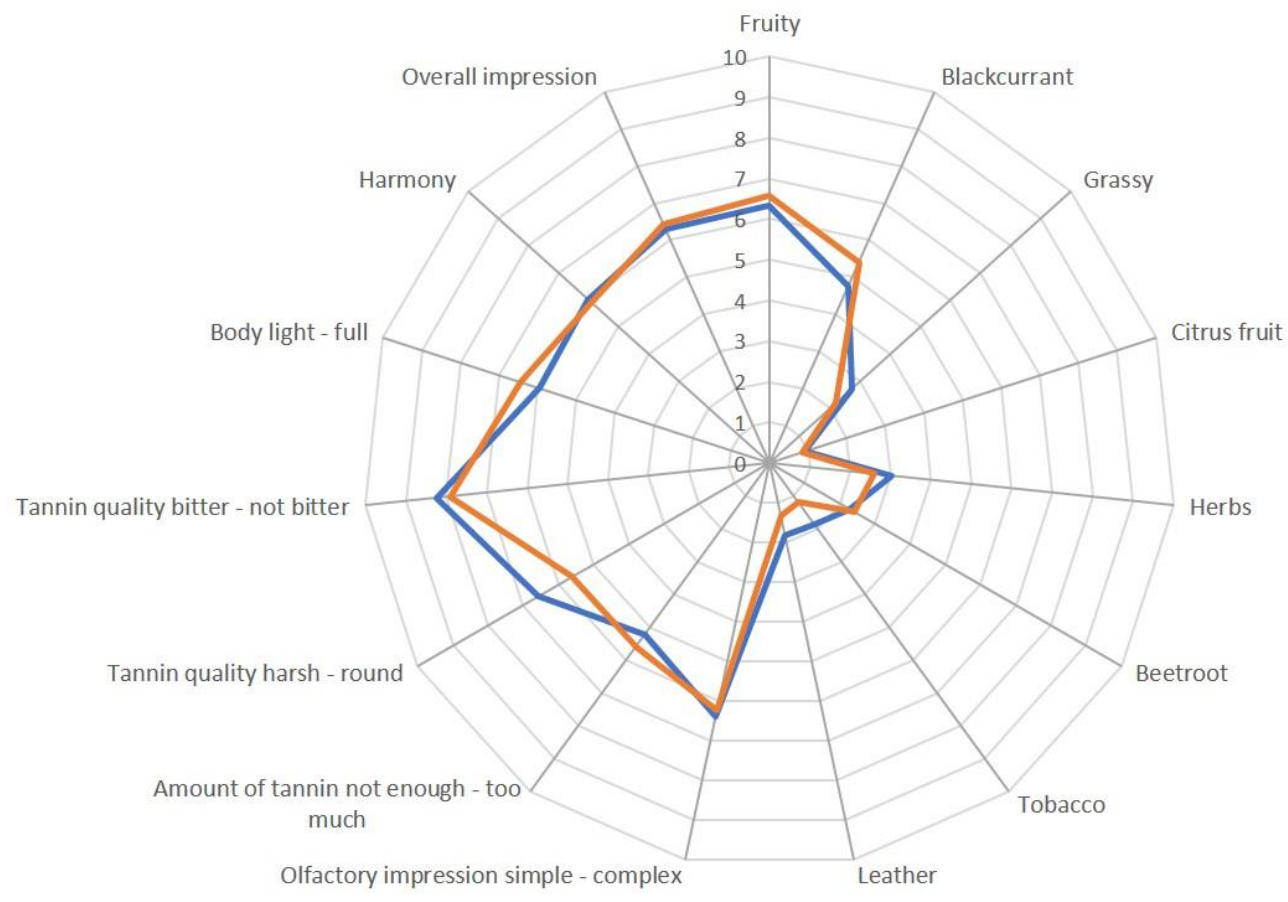

— cool fermentation temperature

—warm fermentation temperature

Abb. 3: Spinnennetzdiagramm zur sensorischen Beschreibung Jahrgang 2012 und 2013; Vergleich aller Varianten kühle Gärtemperatur und warme Gärtemperatur. ${ }^{*} p \leq 0.05 ;{ }^{* *} p \leq 0.01 ;{ }^{* *} p \leq 0.001 / /$ Radar chart of the sensorial description vintage 2012 and 2013 ; comparison of all variants cool fermentation temperature and warm fermentation temperature. ${ }^{*} p \leq 0.05 ; * * \leq 0.01 ; * * p \leq 0.001$. 


\section{Comparison: short mash contact time - long mash contact time}

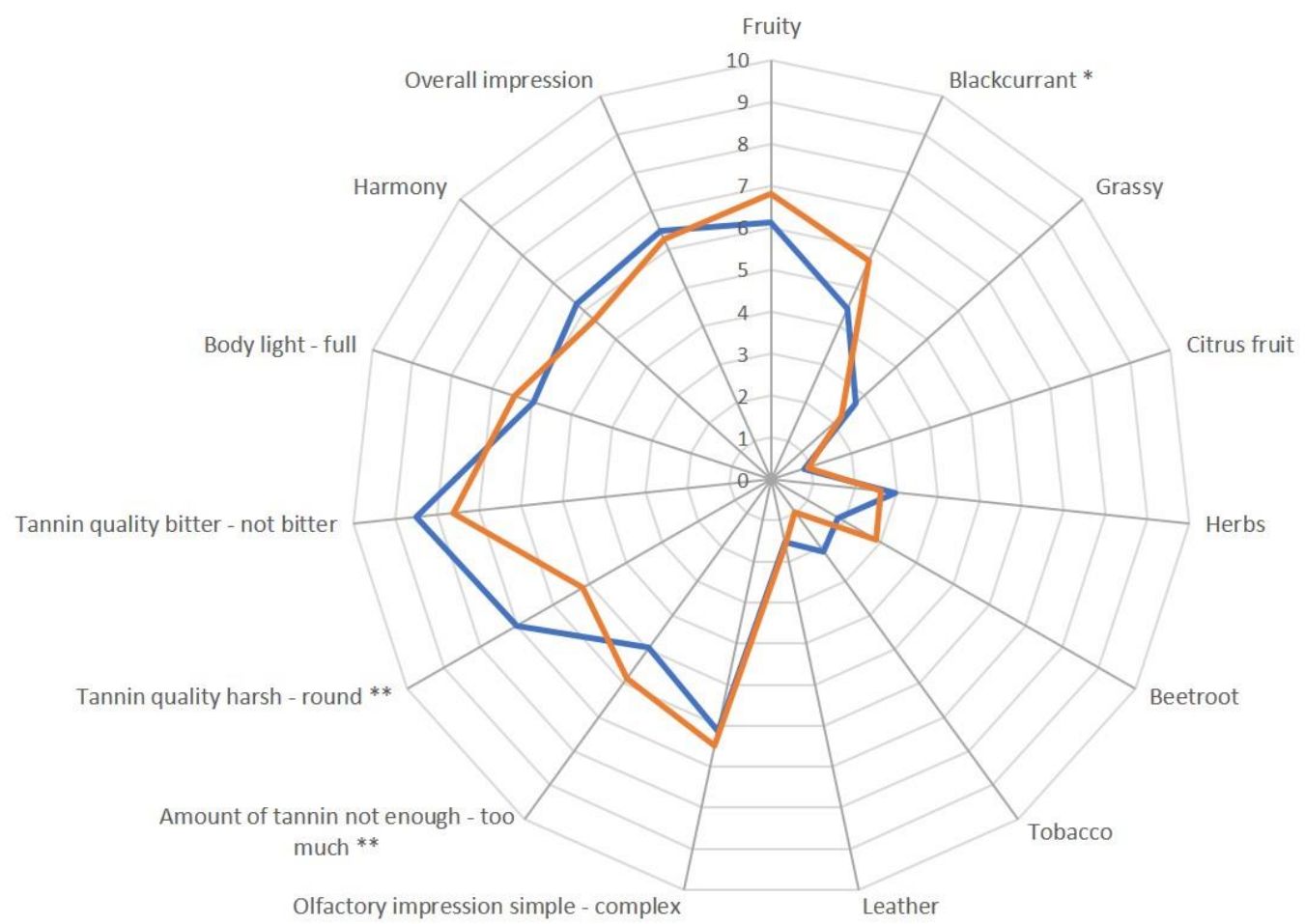

— short mash contact —ong mash contact

Abb. 4: Spinnennetzdiagramm zur sensorischen Beschreibung Jahrgang 2012 und 2013; Vergleich aller Varianten kurze Maischekontaktzeit und lange Maischekontaktzeit. * $\mathrm{p} \leq 0.05 ;{ }^{* *} \mathrm{p} \leq 0.01 ;{ }^{* * *} \mathrm{p} \leq 0.001 / /$ Radar chart of the sensorial description vintage 2012 and 2013; comparison of all variants short mash contact time and long mash contact time. ${ }^{*} p \leq 0.05 ;{ }^{* *} p \leq 0.01 ;{ }^{* * *} p \leq 0.001$. 

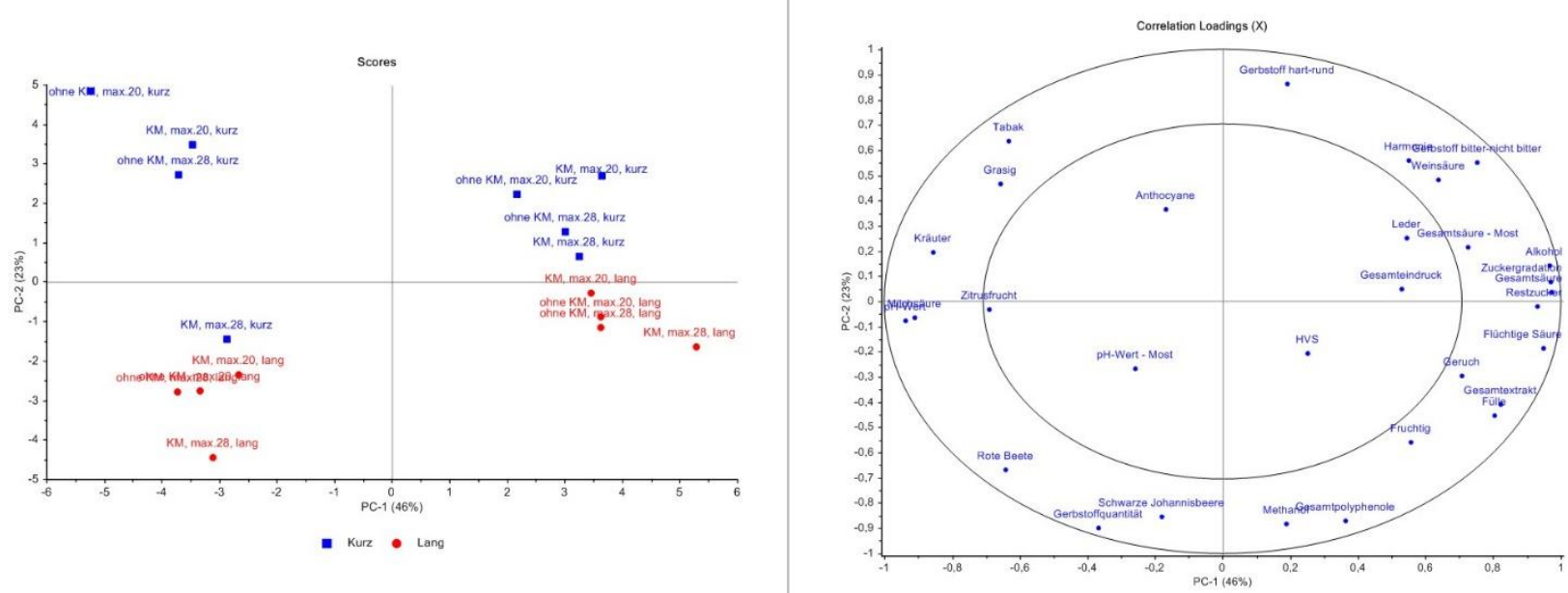

Abb. 5: Eigenwert und Ladungskorrelationendiagramm einer Hauptkomponentenanalyse zur Beschreibung der Weine auf der Basis der gemessenen chemisch-analytischen sowie sensorischen Charakteristiken der untersuchten Varianten der Jahre 2012 und 2013 // Scores and correlation-loadings plot of a principle component analysis based on the chemical-analytical and sensorial characteristics of the wines from the vintage 2012 and 2013. 


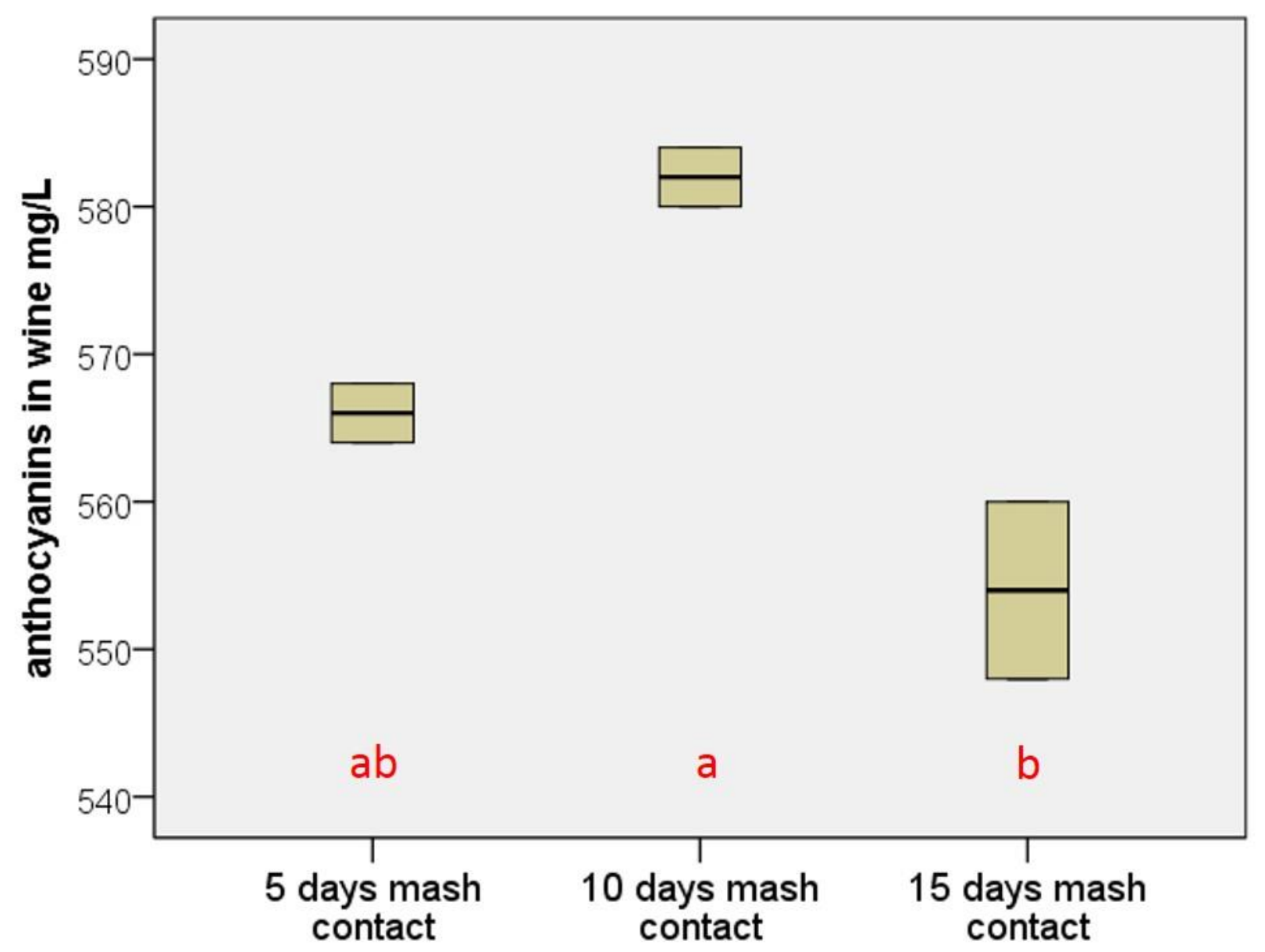

Abb. 6: Boxplot zum analytischen Parameter Anthocyane (* Boxplots welche mit verschiedenen Buchstaben gekennzeichnet sind, unterscheiden sich mit dem Tukey-B Test bei $p \leq 0,05$ signifikant) // Boxplot for the analytical parameter anthocyanins (* boxplots marked with different letters differ significantly with the Tukey-B test at $p \leq 0.05)$. 


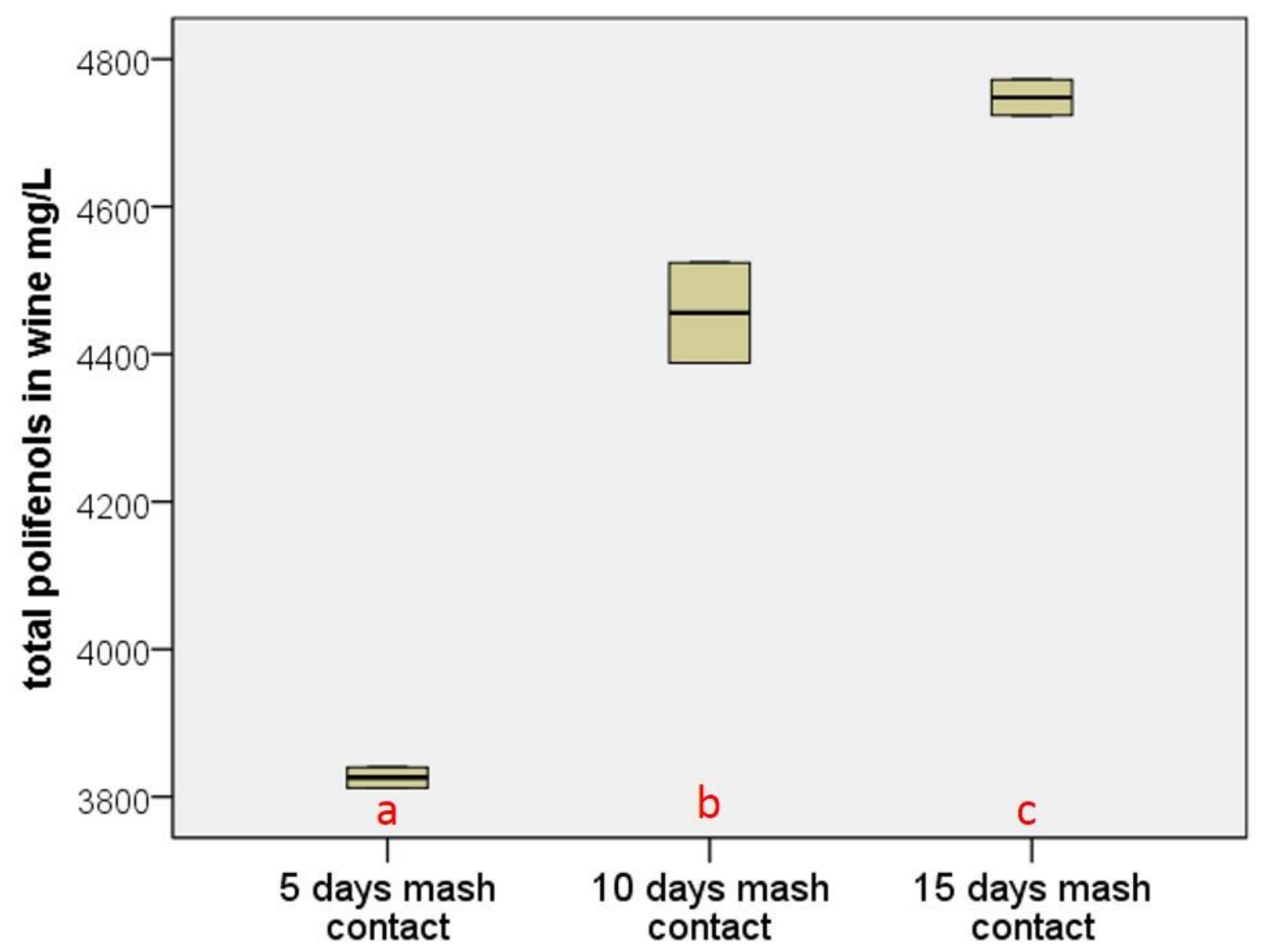

Abb. 7: Boxplot zum analytischen Parameter Gesamtpolyphenole (* Boxplots welche mit verschiedenen Buchstaben gekennzeichnet sind, unterscheiden sich mit dem Tukey-B Test bei $\mathrm{p} \leq 0,05$ signifikant) // Boxplot for the analytical parameter total polifenols (* boxplots marked with different letters differ significantly with the Tukey-B test at $p \leq 0.05$ ). 


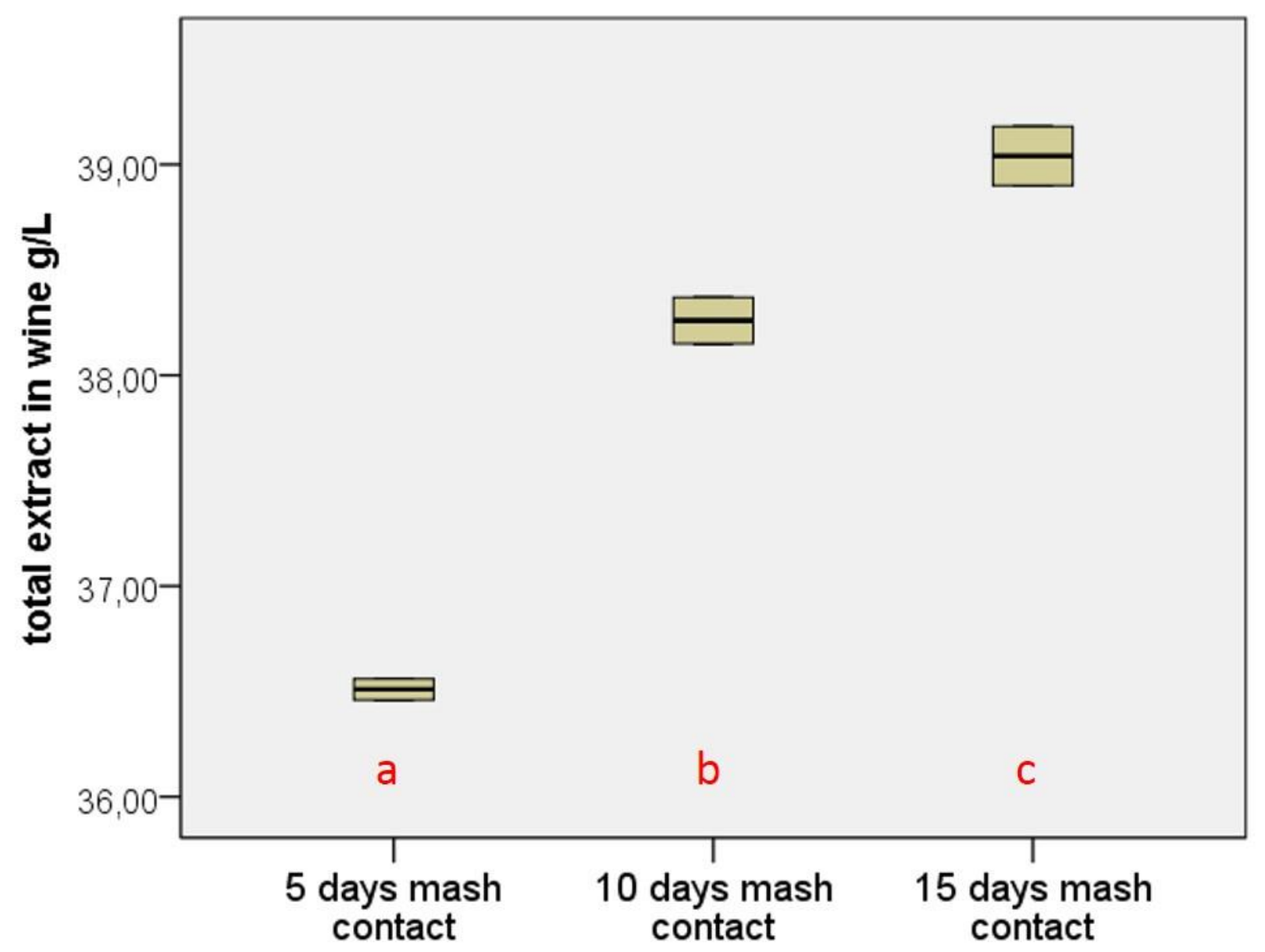

Abb. 8: Boxplot zum analytischen Parameter Gesamtextrakt (* Boxplots welche mit verschiedenen Buchstaben gekennzeichnet sind, unterscheiden sich mit dem Tukey-B Test bei $\mathrm{p} \leq 0,05$ signifikant) // Boxplot for the analytical parameter total extract ( ${ }^{*}$ boxplots marked with different letters differ significantly with the Tukey-B test at $p \leq 0.05$ ). 


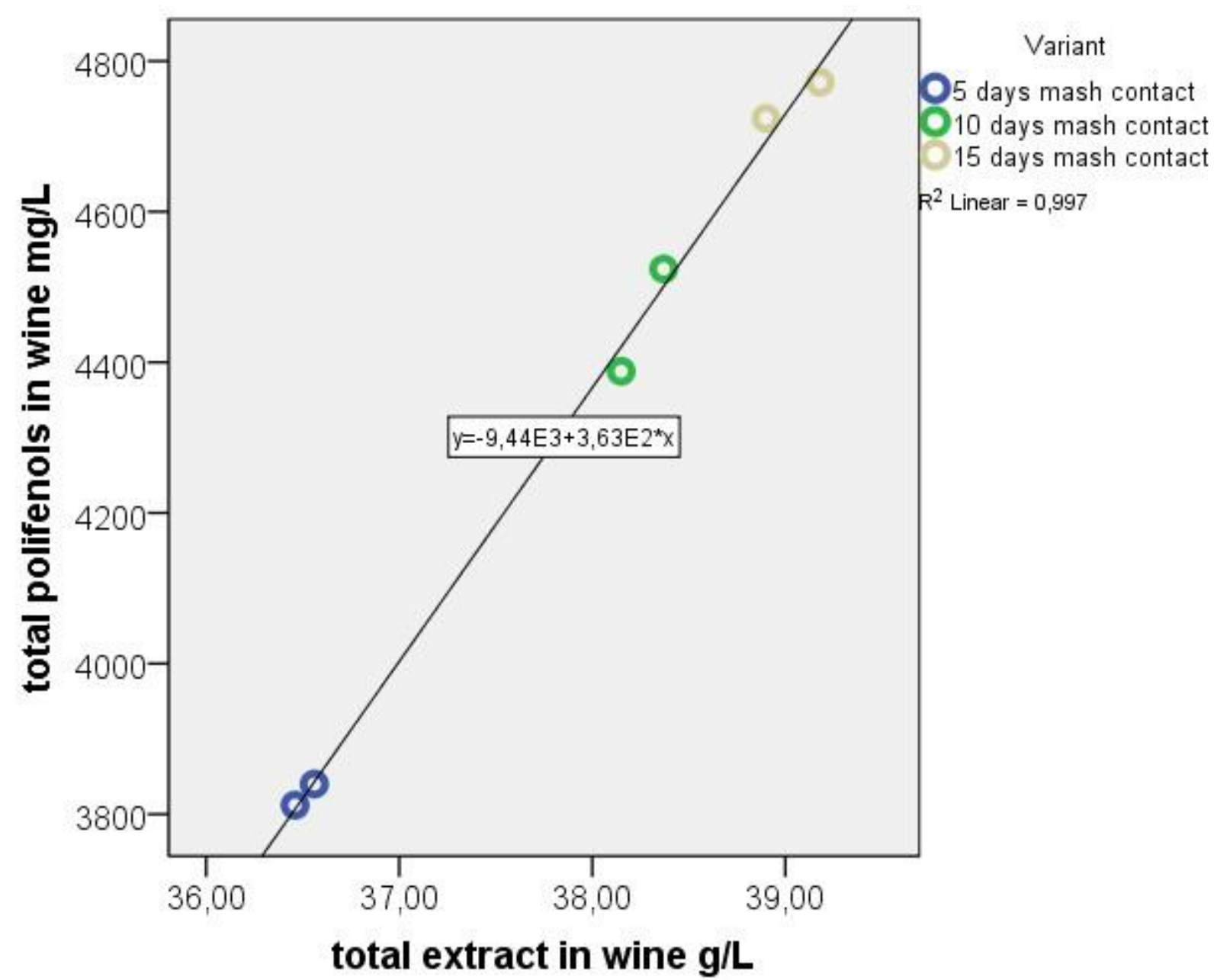

Abb. 9: Einfaches Streudiagramm zwischen dem analystischen Parameter Gesamtpolyphenole und dem analytischen Parameter Gesamtextrakt // Simple scatter plot between the analystic parameter total poliphenols and the analystic parameter total extract. 


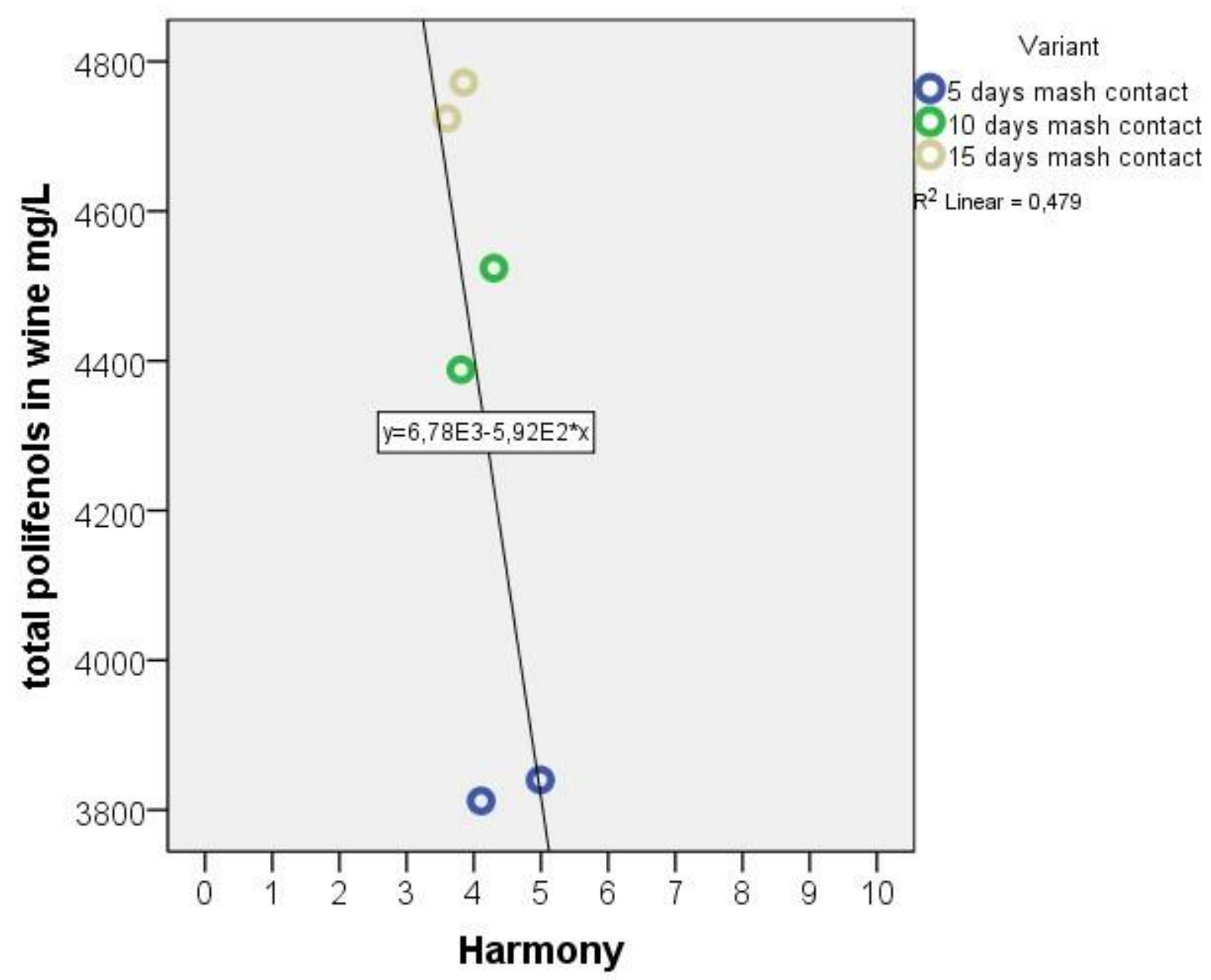

Abb. 10: Einfaches Streudiagramm zwischen dem analystischen Parameter Gesamtpolyphenole und dem sensorischen Parameter Harmonie im Wein // Simple scatter plot between the analystic parameter total poliphenols and the sensory parameter harmony in the wine. 


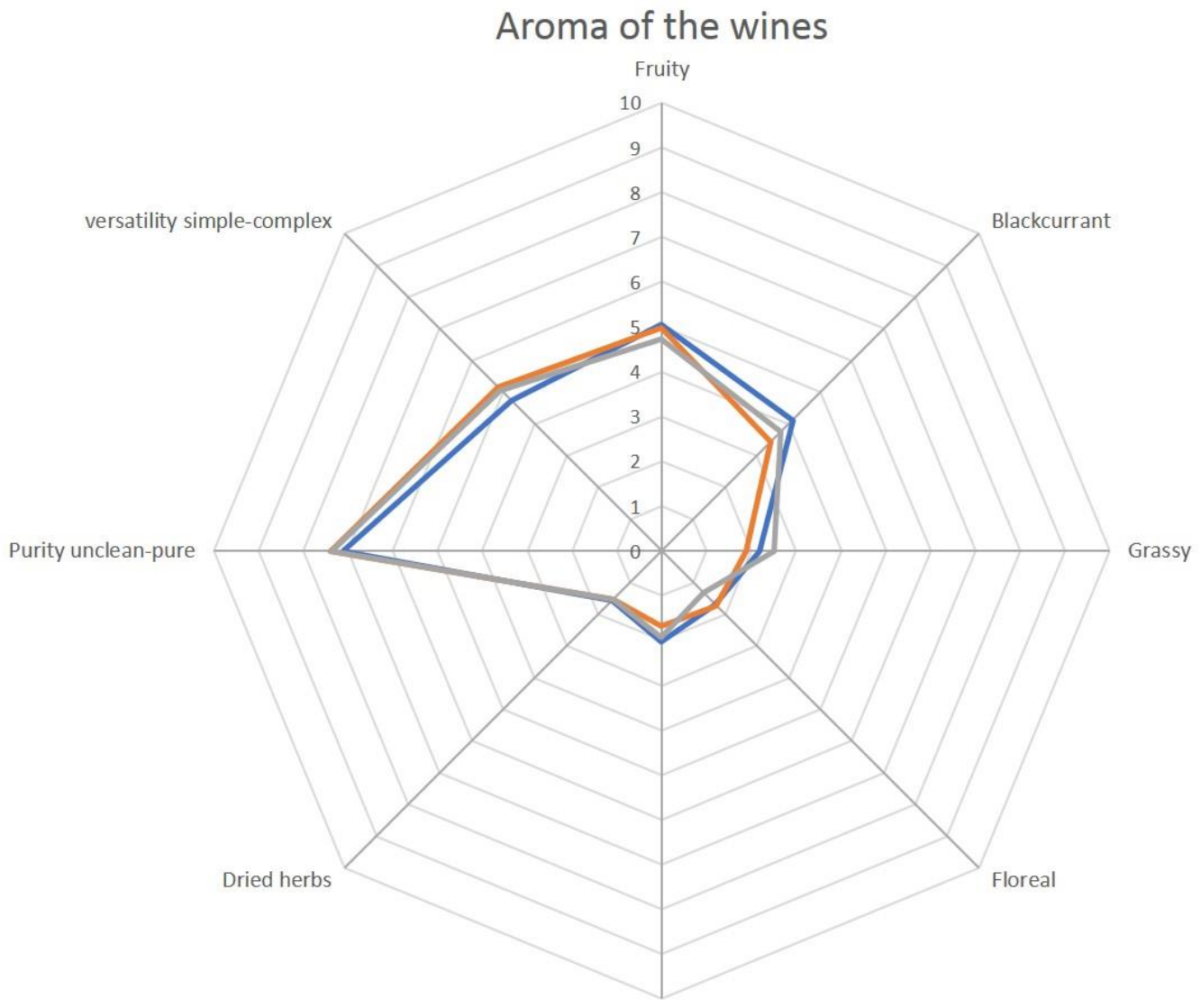

Spicy

-5 days mash contact $\quad-10$ days mash contact $\quad-15$ days mash contact

Abb. 11: Ergebnisse der Sensorik des Jahrganges 2015, Aromen im Wein. * $p \leq 0.05 ;{ }^{* *} p \leq 0.01 ; * * * p \leq 0.001 / /$ Results of the sensory analysis of the year 2015 , aromas in the wine. ${ }^{*} p \leq 0.05 ;{ }^{* *} p \leq 0.01 ;{ }^{* * *} p \leq 0.001$. 


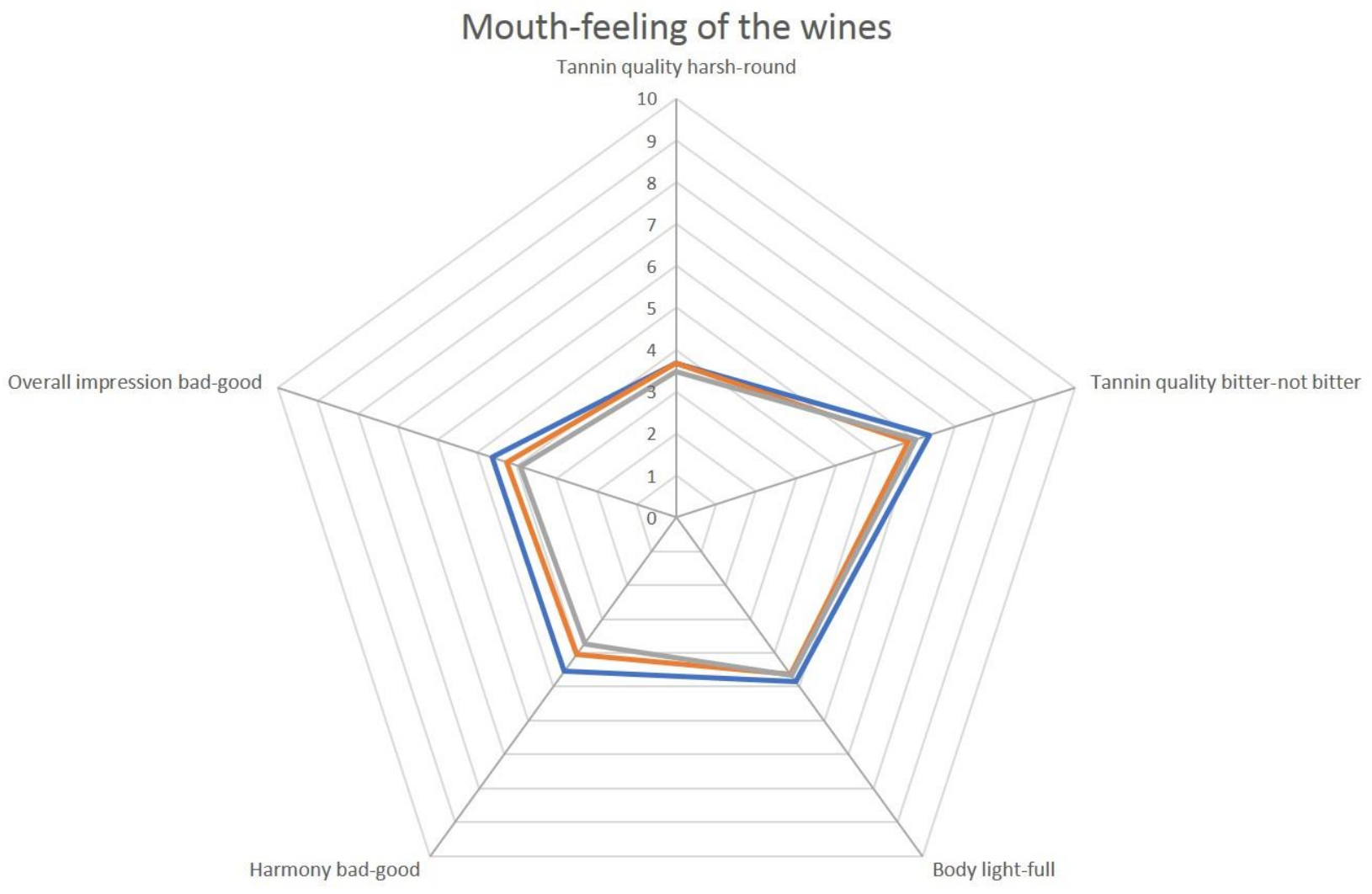

-5 days mash contact $\quad-10$ days mash contact $\quad-15$ days mash contact

Abb. 12: Ergebnisse der Sensorik des Jahrganges 2015, Mundgefühl im Wein. ${ }^{*} p \leq 0.05 ;{ }^{* *} p \leq 0.01 ;{ }^{* * *} p \leq 0.001 / /$ Results of the sensory analysis of the year 2015 , mouth-feeling in the wine. ${ }^{*} p \leq 0.05{ }^{* *} p \leq 0.01 ;{ }^{* * *} p \leq 0.001$. 


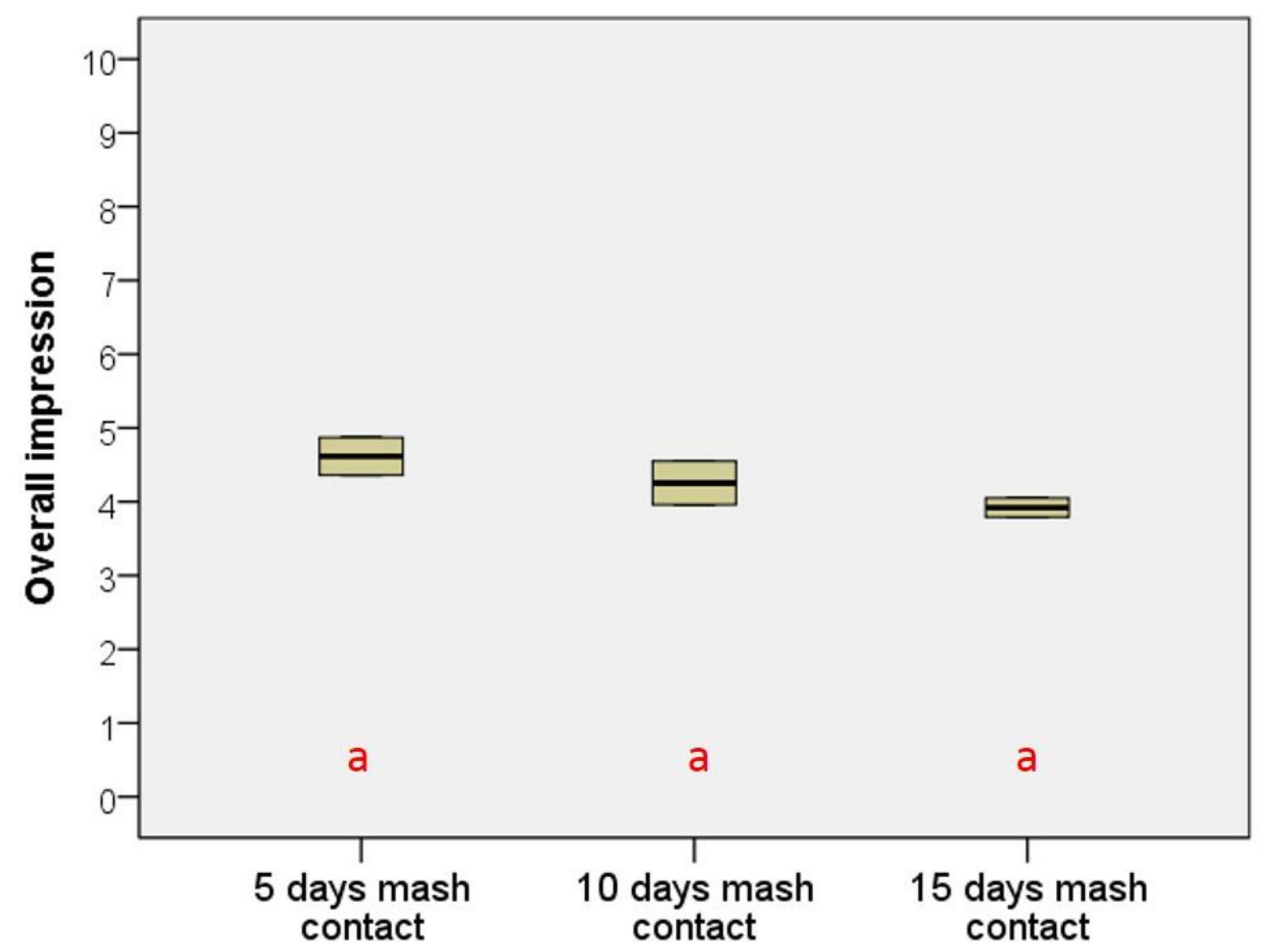

Abb. 13: Boxplot zum sensorischen Parameter Gesamteindruck (* Boxplots welche mit verschiedenen Buchstaben gekennzeichnet sind, unterscheiden sich mit dem Tukey-B Test bei $p \leq 0,05$ signifikant) // Boxplot for the sensorial parameter overall impression (* boxplots marked with different letters differ significantly with the Tukey-B test at $p \leq 0.05)$. 


\section{ANHANG 2: TABELLEN}

Tab. 1: Überblick über die vinifizierten Varianten // Overview of the vinified variants.

Gärtemperatur max. $20^{\circ} \mathrm{C}$, Maischekontakt 4 Tage

Fermentation temperature max. $20^{\circ} \mathrm{C}$, mash contact 4 days

Gärtemperatur max. $20^{\circ} \mathrm{C}$, Maischekontakt Ausgärung

Fermentation temperature max. $20^{\circ} \mathrm{C}$, mash contact end of fermentation

$x \quad x$

Gärtemperatur max. $28^{\circ} \mathrm{C}$, Maischekontakt 4 Tage

Fermentation temperature max. $28^{\circ} \mathrm{C}$, mash contact 4 days

Gärtemperatur max. $28^{\circ} \mathrm{C}$, Maischekontakt Ausgärung

Fermentation temperature max. $28^{\circ} \mathrm{C}$, mash contact end of fermentation

Kaltmazeration 6 Tage bei $4{ }^{\circ} \mathrm{C}$, Gärtemperatur max. $20{ }^{\circ} \mathrm{C}$, Maischekontakt 4 Tage

Cold maceration 6 days at $4{ }^{\circ} \mathrm{C}$, Fermentation temperature max. $20^{\circ} \mathrm{C}$, mash contact 4 days

Kaltmazeration 6 Tage bei $4{ }^{\circ} \mathrm{C}$, Gärtemperatur max. $20^{\circ} \mathrm{C}$, Maischekontakt Ausgärung

Cold maceration 6 days at $4{ }^{\circ} \mathrm{C}$, Fermentation temperature max. $20^{\circ} \mathrm{C}$, mash contact end of fermentation

Kaltmazeration 6 Tage bei $4{ }^{\circ} \mathrm{C}$, Gärtemperatur max. $28^{\circ} \mathrm{C}$, Maischekontakt 4 Tage

Cold maceration 6 days at $4{ }^{\circ} \mathrm{C}$, Fermentation temperature max. $28^{\circ} \mathrm{C}$, mash contact 4 days

Kaltmazeration 6 Tage bei $4{ }^{\circ} \mathrm{C}$, Gärtemperatur max. $28^{\circ} \mathrm{C}$, Maischekontakt Ausgärung

Cold maceration 6 days at $4{ }^{\circ} \mathrm{C}$, Fermentation temperature max. $28^{\circ} \mathrm{C}$, mash contact end of fermentation

Mostgärung Rosè

Must fermentation Rosè

Maischekontakt 5 Tage

Mash contact 5 days

Maischekontakt 10 Tage

Mash contact 10 days

Maischekontakt 15 Tage

Mash contact 15 days 
Tab. 2: Mostanalysen der Ausgangsmaischen // Must analyzes of the grape material.

\begin{tabular}{cccccc}
\hline $\begin{array}{l}\text { Jahr } \\
\text { year }\end{array}$ & $\begin{array}{c}\text { Erntedatum } \\
\text { harvest date }\end{array}$ & $\begin{array}{c}\text { Herkunft } \\
\text { origin }\end{array}$ & $\begin{array}{c}\text { Mostgewicht }{ }^{\circ} \mathrm{KMW} \\
\text { total soluble solids ( }{ }^{\circ} \mathrm{KMW} \text { ) }\end{array}$ & $\begin{array}{c}\text { pH-Wert } \\
\text { Most } \\
\text { pH must }\end{array}$ & $\begin{array}{c}\text { Gesamtsäure im Most g/l } \\
\text { titratable acidity in must g/l }\end{array}$ \\
\hline $\mathbf{2 0 1 2}$ & 19.09 .2012 & Piglon (Pfatten; Südtirol) & 22,52 & 3,49 & 5,91 \\
\hline $\mathbf{2 0 1 3}$ & 03.10 .2013 & Piglon (Pfatten; Südtirol) & 21,20 & 3,40 & 5,87 \\
\hline $\mathbf{2 0 1 5}$ & 15.09 .2015 & Piglon (Pfatten; Südtirol) & 22,52 & 3,49 & 5,45
\end{tabular}


Tab. 3: Verkostete Parameter 2012 // Tasted parameters 2012.

\begin{tabular}{|c|c|}
\hline $\begin{array}{l}\text { Kriterium } \\
\text { criteria }\end{array}$ & $\begin{array}{l}\text { Beschreibung } \\
\text { description }\end{array}$ \\
\hline $\begin{array}{l}\text { Fruchtig } \\
\text { Fruity }\end{array}$ & $\begin{array}{l}\text { nicht vorhanden - vorhanden } \\
\text { not present - present }\end{array}$ \\
\hline $\begin{array}{c}\text { Schwarze Johannisbeere } \\
\text { Blackcurrant }\end{array}$ & $\begin{array}{l}\text { nicht vorhanden - vorhanden } \\
\text { not present - present }\end{array}$ \\
\hline $\begin{array}{l}\text { Grasig } \\
\text { Grassy }\end{array}$ & $\begin{array}{l}\text { nicht vorhanden - vorhanden } \\
\text { not present - present }\end{array}$ \\
\hline $\begin{array}{l}\text { Zitrusfrucht } \\
\text { Citrus fruit }\end{array}$ & $\begin{array}{l}\text { nicht vorhanden - vorhanden } \\
\text { not present - present }\end{array}$ \\
\hline $\begin{array}{l}\text { Kräuter } \\
\text { Herbs }\end{array}$ & $\begin{array}{l}\text { nicht vorhanden - vorhanden } \\
\text { not present - present }\end{array}$ \\
\hline $\begin{array}{l}\text { Rote Beete } \\
\text { Beetroot }\end{array}$ & $\begin{array}{l}\text { nicht vorhanden - vorhanden } \\
\text { not present - present }\end{array}$ \\
\hline $\begin{array}{l}\text { Tabak } \\
\text { Tobacco }\end{array}$ & $\begin{array}{l}\text { nicht vorhanden - vorhanden } \\
\text { not present - present }\end{array}$ \\
\hline $\begin{array}{l}\text { Leder } \\
\text { Leather }\end{array}$ & $\begin{array}{l}\text { nicht vorhanden - vorhanden } \\
\text { not present - present }\end{array}$ \\
\hline $\begin{array}{l}\text { Tee } \\
\text { Tea }\end{array}$ & $\begin{array}{l}\text { nicht vorhanden - vorhanden } \\
\text { not present - present }\end{array}$ \\
\hline $\begin{array}{l}\text { Marmelade } \\
\quad \text { jam }\end{array}$ & $\begin{array}{l}\text { nicht vorhanden - vorhanden } \\
\text { not present - present }\end{array}$ \\
\hline $\begin{array}{l}\text { Gewürze } \\
\text { spices }\end{array}$ & $\begin{array}{l}\text { nicht vorhanden - vorhanden } \\
\text { not present - present }\end{array}$ \\
\hline $\begin{array}{c}\text { Geruch } \\
\text { Olfactory impression }\end{array}$ & $\begin{array}{l}\text { einfach - vielseitig } \\
\text { simple - complex }\end{array}$ \\
\hline $\begin{array}{l}\text { Gerbstoffquantität } \\
\text { Amount of tannin }\end{array}$ & $\begin{array}{c}\text { zu wenig - zu viel } \\
\text { not enough - too much }\end{array}$ \\
\hline $\begin{array}{l}\text { Gerbstoff hart } \\
\text { Tannin quality }\end{array}$ & $\begin{array}{l}\text { hart - rund } \\
\text { harsh - round }\end{array}$ \\
\hline $\begin{array}{l}\text { Gerbstoff bitter } \\
\text { Tannin quality }\end{array}$ & $\begin{array}{l}\text { bitter - nicht bitter } \\
\text { bitter - not bitter }\end{array}$ \\
\hline $\begin{array}{l}\text { Fülle } \\
\text { Body }\end{array}$ & $\begin{array}{l}\text { dünn - voll } \\
\text { light - full }\end{array}$ \\
\hline $\begin{array}{l}\text { Harmonie } \\
\text { Harmony }\end{array}$ & $\begin{array}{l}\text { schlecht - gut } \\
\text { bad-good }\end{array}$ \\
\hline $\begin{array}{l}\text { Gesamteindruck } \\
\text { Overall impression }\end{array}$ & $\begin{array}{l}\text { schlecht - gut } \\
\text { bad-good }\end{array}$ \\
\hline
\end{tabular}


Tab. 4: Verkostete Parameter 2013 // Tasted parameters 2013.

\begin{tabular}{|c|c|}
\hline $\begin{array}{l}\text { Kriterium } \\
\text { criteria }\end{array}$ & $\begin{array}{l}\text { Beschreibung } \\
\text { description }\end{array}$ \\
\hline $\begin{array}{l}\text { Fruchtig } \\
\text { Fruity }\end{array}$ & $\begin{array}{l}\text { nicht vorhanden - vorhanden } \\
\text { not present - present }\end{array}$ \\
\hline $\begin{array}{c}\text { Schwarze Johannisbeere } \\
\text { Blackcurrant }\end{array}$ & $\begin{array}{c}\text { nicht vorhanden - vorhanden } \\
\text { not present - present }\end{array}$ \\
\hline $\begin{array}{l}\text { Grasig } \\
\text { Grassy }\end{array}$ & $\begin{array}{c}\text { nicht vorhanden - vorhanden } \\
\text { not present - present }\end{array}$ \\
\hline $\begin{array}{l}\text { Zitrusfrucht } \\
\text { Citrus fruit }\end{array}$ & $\begin{array}{l}\text { nicht vorhanden - vorhanden } \\
\text { not present - present }\end{array}$ \\
\hline $\begin{array}{l}\text { Kräuter } \\
\text { Herbs }\end{array}$ & $\begin{array}{c}\text { nicht vorhanden - vorhanden } \\
\text { not present - present }\end{array}$ \\
\hline $\begin{array}{l}\text { Rote Beete } \\
\text { Beetroot }\end{array}$ & $\begin{array}{l}\text { nicht vorhanden - vorhanden } \\
\text { not present - present }\end{array}$ \\
\hline $\begin{array}{l}\text { Tabak } \\
\text { Tobacco }\end{array}$ & $\begin{array}{c}\text { nicht vorhanden - vorhanden } \\
\text { not present - present }\end{array}$ \\
\hline $\begin{array}{l}\text { Leder } \\
\text { Leather }\end{array}$ & $\begin{array}{l}\text { nicht vorhanden - vorhanden } \\
\text { not present - present }\end{array}$ \\
\hline $\begin{array}{c}\text { Geruch } \\
\text { Olfactory impression }\end{array}$ & $\begin{array}{l}\text { einfach - vielseitig } \\
\text { simple - complex }\end{array}$ \\
\hline $\begin{array}{l}\text { Gerbstoffquantität } \\
\text { Amount of tannin }\end{array}$ & $\begin{array}{l}\text { zu wenig - zu viel } \\
\text { not enough - too much }\end{array}$ \\
\hline $\begin{array}{l}\text { Gerbstoff hart } \\
\text { Tannin quality }\end{array}$ & $\begin{array}{l}\text { hart - rund } \\
\text { harsh - round }\end{array}$ \\
\hline $\begin{array}{l}\text { Gerbstoff bitter } \\
\text { Tannin quality }\end{array}$ & $\begin{array}{l}\text { bitter - nicht bitter } \\
\text { bitter - not bitter }\end{array}$ \\
\hline $\begin{array}{l}\text { Fülle } \\
\text { Body }\end{array}$ & $\begin{array}{l}\text { dünn - voll } \\
\text { light - full }\end{array}$ \\
\hline $\begin{array}{l}\text { Harmonie } \\
\text { Harmony }\end{array}$ & $\begin{array}{l}\text { schlecht - gut } \\
\text { bad-good }\end{array}$ \\
\hline $\begin{array}{l}\text { Gesamteindruck } \\
\text { Overall impression }\end{array}$ & $\begin{array}{l}\text { schlecht - gut } \\
\text { bad-good }\end{array}$ \\
\hline
\end{tabular}


Tab. 5: Verkostete Parameter 2015 // Tasted parameters 2015.

\begin{tabular}{|c|c|}
\hline $\begin{array}{l}\text { Kriterium } \\
\text { criteria }\end{array}$ & $\begin{array}{l}\text { Beschreibung } \\
\text { description }\end{array}$ \\
\hline $\begin{array}{l}\text { Fruchtig } \\
\text { Fruity }\end{array}$ & $\begin{array}{l}\text { nicht vorhanden - vorhanden } \\
\text { not present - present }\end{array}$ \\
\hline $\begin{array}{c}\text { Schwarze Johannisbeere } \\
\text { Blackcurrant }\end{array}$ & $\begin{array}{l}\text { nicht vorhanden - vorhanden } \\
\text { not present - present }\end{array}$ \\
\hline $\begin{array}{l}\text { Grüne Noten } \\
\text { Grassy }\end{array}$ & $\begin{array}{l}\text { nicht vorhanden - vorhanden } \\
\text { not present - present }\end{array}$ \\
\hline $\begin{array}{l}\text { Floreal } \\
\text { Floreal }\end{array}$ & $\begin{array}{l}\text { nicht vorhanden - vorhanden } \\
\text { not present - present }\end{array}$ \\
\hline $\begin{array}{l}\text { Würzig } \\
\text { Spicy }\end{array}$ & $\begin{array}{l}\text { nicht vorhanden - vorhanden } \\
\text { not present - present }\end{array}$ \\
\hline $\begin{array}{l}\text { Getrocknete Kräuter } \\
\text { Dried herbs }\end{array}$ & $\begin{array}{l}\text { nicht vorhanden - vorhanden } \\
\text { not present - present }\end{array}$ \\
\hline $\begin{array}{l}\text { Reintönigkeit } \\
\text { Purity }\end{array}$ & $\begin{array}{l}\text { unsauber - sauber } \\
\text { unclean - pure }\end{array}$ \\
\hline $\begin{array}{l}\text { Vielseitigkeit } \\
\text { versatility }\end{array}$ & $\begin{array}{l}\text { einfach - vielseitig } \\
\text { simple - complex }\end{array}$ \\
\hline $\begin{array}{l}\text { Gerbstoffquantität } \\
\text { Amount of tannin }\end{array}$ & $\begin{array}{l}\text { zu wenig - zu viel } \\
\text { not enough - too much }\end{array}$ \\
\hline $\begin{array}{l}\text { Gerbstoff hart } \\
\text { Tannin quality }\end{array}$ & $\begin{array}{l}\text { hart - rund } \\
\text { harsh - round }\end{array}$ \\
\hline $\begin{array}{l}\text { Gerbstoff bitter } \\
\text { Tannin quality }\end{array}$ & $\begin{array}{l}\text { bitter - nicht bitter } \\
\text { bitter - not bitter }\end{array}$ \\
\hline $\begin{array}{l}\text { Fülle } \\
\text { Body }\end{array}$ & $\begin{array}{l}\text { dünn - voll } \\
\text { light - full }\end{array}$ \\
\hline $\begin{array}{l}\text { Harmonie } \\
\text { Harmony }\end{array}$ & $\begin{array}{l}\text { schlecht - gut } \\
\text { bad-good }\end{array}$ \\
\hline $\begin{array}{l}\text { Gesamteindruck } \\
\text { Overall impression }\end{array}$ & $\begin{array}{l}\text { schlecht - gut } \\
\text { bad-good }\end{array}$ \\
\hline
\end{tabular}


Tab. 6: Deskriptive Statistik Weinanalysen, 5 Tage Maischegärung // Descriptive statistics Wine analysis, 5 days mash fermentation.

\begin{tabular}{|c|c|c|c|c|c|}
\hline & $\mathbf{N}$ & Minimum & Maximum & Average & Standard deviation \\
\hline $\begin{array}{l}\text { Alkohol im Wein \% vol } \\
\text { alcohol in wine \% vol }\end{array}$ & 2 & 15,08 & 15,21 & 15,145 & 0,091923882 \\
\hline $\begin{array}{l}\mathrm{pH}-\text { Wert im Wein } \\
\mathrm{pH} \text { in wine }\end{array}$ & 2 & 3,87 & 3,87 & 3,87 & 0 \\
\hline $\begin{array}{l}\text { Gesamtsäure im Wein } \mathrm{g} / \mathrm{l} \\
\text { titratable acidity wine } \mathrm{g} / \mathrm{l}\end{array}$ & 2 & 5,33 & 5,46 & 5,395 & 0,091923882 \\
\hline $\begin{array}{l}\text { Anthocyane im Wein } \mathrm{mg} / \mathrm{l} \\
\text { anthocyanins in wine } \mathrm{mg} / \mathrm{l}\end{array}$ & 2 & 564 & 568 & 566 & 2,828427125 \\
\hline $\begin{array}{l}\text { Gesamtpolyphenole im Wein } \mathrm{mg} / \mathrm{l} \\
\text { total polifenols in wine } \mathrm{mg} / \mathrm{l}\end{array}$ & 2 & 3812 & 3840 & 3826 & 19,79898987 \\
\hline $\begin{array}{l}\text { Gesamtextrakt im Wein } \mathrm{g} / \mathrm{l} \\
\text { total extract in wine } \mathrm{g} / \mathrm{l}\end{array}$ & 2 & 36,46 & 36,56 & 36,51 & 0,070710678 \\
\hline $\begin{array}{l}\text { Weinsäure im Wein } \mathrm{g} / \mathrm{l} \\
\text { tartaric acid in wine } \mathrm{g} / \mathrm{l}\end{array}$ & 2 & 1,66 & 1,72 & 1,69 & 0,042426407 \\
\hline $\begin{array}{l}\text { Milchsäure im Wein } \mathrm{g} / \mathrm{l} \\
\text { lactic acid in wine } \mathrm{g} / \mathrm{l}\end{array}$ & 2 & 1,54 & 1,55 & 1,545 & 0,007071068 \\
\hline $\begin{array}{l}\text { Flüchtige Säure im Wein g/l } \\
\text { volatile acid in wine } \mathrm{g} / \mathrm{l}\end{array}$ & 2 & 0,63 & 0,63 & 0,63 & 0 \\
\hline $\begin{array}{l}\text { Methanol im Wein } \mathrm{g} / \mathrm{l} \\
\text { methanol in wine } \mathrm{g} / \mathrm{l}\end{array}$ & 2 & 0,13 & 0,14 & 0,135 & 0,007071068 \\
\hline $\begin{array}{c}\text { Gültige Werte (Listenweise) } \\
\text { valid values (listwise) }\end{array}$ & 2 & & & & \\
\hline
\end{tabular}


Tab. 7: Deskriptive Statistik Weinanalysen Variante 10 Tage Maischegärung // Descriptive statistics Wine analysis, 10 days mash fermentation.

\begin{tabular}{|c|c|c|c|c|c|}
\hline & $\mathbf{N}$ & Minimum & Maximum & Average & Standard deviation \\
\hline $\begin{array}{l}\text { Alkohol im Wein \% vol } \\
\text { alcohol in wine \% vol }\end{array}$ & 2 & 14,94 & 15,07 & 15,005 & 0,091923882 \\
\hline $\begin{array}{l}\mathrm{pH} \text {-Wert im Wein } \\
\text { pH in wine }\end{array}$ & 2 & 3,89 & 3,91 & 3,90 & 0,014142136 \\
\hline $\begin{array}{l}\text { Gesamtsäure im Wein } \mathrm{g} / \mathrm{l} \\
\text { titratable acidity wine } \mathrm{g} / \mathrm{l}\end{array}$ & 2 & 5,29 & 5,47 & 5,38 & 0,127279221 \\
\hline $\begin{array}{l}\text { Anthocyane im Wein } \mathrm{mg} / \mathrm{l} \\
\text { anthocyanins in wine } \mathrm{mg} / \mathrm{l}\end{array}$ & 2 & 580 & 584 & 582 & 2,828427125 \\
\hline $\begin{array}{l}\text { Gesamtpolyphenole im Wein } \mathrm{mg} / \mathrm{l} \\
\text { total polifenols in wine } \mathrm{mg} / \mathrm{l}\end{array}$ & 2 & 4388 & 4524 & 4456 & 96,16652224 \\
\hline $\begin{array}{l}\text { Gesamtextrakt im Wein } \mathrm{g} / \mathrm{l} \\
\text { total extract in wine } \mathrm{g} / \mathrm{l}\end{array}$ & 2 & 38,15 & 38,37 & 38,26 & 0,155563492 \\
\hline $\begin{array}{l}\text { Weinsäure im Wein } \mathrm{g} / \mathrm{l} \\
\text { tartaric acid in wine } \mathrm{g} / \mathrm{l}\end{array}$ & 2 & 1,63 & 1,65 & 1,64 & 0,014142136 \\
\hline $\begin{array}{l}\text { Milchsäure im Wein g/l } \\
\text { lactic acid in wine } \mathrm{g} / \mathrm{l}\end{array}$ & 2 & 1,49 & 1,60 & 1,545 & 0,077781746 \\
\hline $\begin{array}{l}\text { Flüchtige Säure im Wein } \mathrm{g} / \mathrm{l} \\
\text { volatile acid in wine } \mathrm{g} / \mathrm{l}\end{array}$ & 2 & 0,63 & 0,64 & 0,635 & 0,007071068 \\
\hline $\begin{array}{l}\text { Methanol im Wein } \mathrm{g} / \mathrm{l} \\
\text { methanol in wine } \mathrm{g} / \mathrm{l}\end{array}$ & 2 & 0,15 & 0,15 & 0,15 & 0 \\
\hline $\begin{array}{l}\text { Gültige Werte (Listenweise) } \\
\text { valid values (listwise) }\end{array}$ & 2 & & & & \\
\hline
\end{tabular}


Tab. 8: Deskriptive Statistik Weinanalysen Variante 15 Tage Maischegärung // Descriptive statistics Wine analysis, 15 days mash fermentation.

\begin{tabular}{|c|c|c|c|c|c|}
\hline & $\mathbf{N}$ & Minimum & Maximum & Average & Standard deviation \\
\hline $\begin{array}{l}\text { Alkohol im Wein \% vol } \\
\text { alcohol in wine \% vol }\end{array}$ & 2 & 14,92 & 14,98 & 14,95 & 0,042426407 \\
\hline $\begin{array}{l}\mathrm{pH} \text {-Wert im Wein } \\
\mathrm{pH} \text { in wine }\end{array}$ & 2 & 3,92 & 3,95 & 3,935 & 0,021213203 \\
\hline $\begin{array}{l}\text { Gesamtsäure im Wein } \mathrm{g} / \mathrm{l} \\
\text { titratable acidity wine } \mathrm{g} / \mathrm{l}\end{array}$ & 2 & 5,31 & 5,31 & 5,31 & 0 \\
\hline $\begin{array}{l}\text { Anthocyane im Wein } \mathrm{mg} / \mathrm{l} \\
\text { anthocyanins in wine } \mathrm{mg} / \mathrm{l}\end{array}$ & 2 & 548 & 560 & 554 & 8,485281374 \\
\hline $\begin{array}{l}\text { Gesamtpolyphenole im Wein } \mathrm{mg} / \mathrm{l} \\
\text { total polifenols in wine } \mathrm{mg} / \mathrm{l}\end{array}$ & 2 & 4724 & 4772 & 4748 & 33,9411255 \\
\hline $\begin{array}{l}\text { Gesamtextrakt im Wein } \mathrm{g} / \mathrm{l} \\
\text { total extract in wine } \mathrm{g} / \mathrm{l}\end{array}$ & 2 & 38,90 & 39,18 & 39,04 & 0,197989899 \\
\hline $\begin{array}{l}\text { Weinsäure im Wein } \mathrm{g} / \mathrm{l} \\
\text { tartaric acid in wine } \mathrm{g} / \mathrm{l}\end{array}$ & 2 & 1,65 & 1,65 & 1,65 & 0 \\
\hline $\begin{array}{l}\text { Milchsäure im Wein g/l } \\
\text { lactic acid in wine g/l }\end{array}$ & 2 & 1,56 & 1,62 & 1,59 & 0,042426407 \\
\hline $\begin{array}{c}\text { Flüchtige Säure im Wein } \mathrm{g} / \mathrm{l} \\
\text { volatile acid in wine } \mathrm{g} / \mathrm{l}\end{array}$ & 2 & 0,69 & 0,69 & 0,69 & 0 \\
\hline $\begin{array}{l}\text { Methanol im Wein } \mathrm{g} / \mathrm{l} \\
\text { methanol in wine } \mathrm{g} / \mathrm{l}\end{array}$ & 2 & 0,17 & 0,17 & 0,17 & 0 \\
\hline $\begin{array}{c}\text { Gültige Werte (Listenweise) } \\
\text { valid values (listwise) }\end{array}$ & 2 & & & & \\
\hline
\end{tabular}

Für alle Abbildungen und Tabellen ohne Nennung des Urhebers gilt: (C) Versuchszentrum Laimburg. Per tutte le immagini e tabelle senza menzione dell'artefice vale: (C) Centro di Sperimentazione Laimburg. For all figures and tables without mention of the originator applies: (c) Laimburg Research Centre. 